\title{
Recovery of the Decorin-Enriched Fraction, Extract (D), From Human Skin: An Accelerated Protocol
}

\author{
Denys N. Wheatley, ${ }^{1 *}$ Emma Graham, ${ }^{1}$ R. Shannon McMaster, ${ }^{1}$ Ian F. K. Muir, ${ }^{2}$ \\ John D. Holmes, ${ }^{2}$ and Michaela Davies ${ }^{2}$ \\ ${ }^{1}$ BioMedES, MG7, Hilton Campus, Aberdeen AB24 4FA, UK \\ ${ }^{2}$ Plastic Surgery Department, Grampian University Hospitals Trust, Foresterhill, Aberdeen AB25 2ZD, UK
}

Received 10 September 2003; revised 23 February 2004; accepted 3 March 2004

\begin{abstract}
The original extraction procedure of Engel and Catchpole [1] has often been used to recover decorin-enriched material from the skin. This material has a strong inhibitory effect on fibroblast proliferation, and clearly suppresses it in skin except after the first 5-6 days of wounding when new scaffold material is required. The aim of our present study has been to find and evaluate the product of a faster recovery method, and to check its consistency as a more reliable means of regularly obtaining sufficient material for topical application in wounds that might become hypertrophic. Modifications of the original Toole and Lowther [2] extraction procedure have been carefully evaluated in an attempt to cut preparation time without compromising biological activity of the inhibitory extract. We have devised a faster recovery procedure without compromising biological activity, even if initial recovery has been somewhat reduced. The latter problem could be offset by repeated cycles of the final extraction step. The main inhibitory activity is shown to be within the decorin-enriched "extract D," as the core protein and DSPG II. Adjustment of the extract towards neutrality after dialysis against water keeps most of the extracted protein in solution and yielded a decorin-enriched preparation that had a specific activity equivalent to that of the old method. It also yielded a fraction that was readily lyophilised to give a small amount of material that could be stored indefinitely without loss of activity and readily redissolved in aqueous solution. A reliable and relatively quick method is presented for the production, from human skin, of a decorin-enriched preparation that has strong fibroblast inhibitory action. The value of the procedure is that it is inexpensive and can produce the quantities that might be used topically in reducing hypertrophic scarring of wounds.
\end{abstract}

\section{BACKGROUND}

Our aim is to extract the natural inhibitor of fibroblast proliferation, decorin, from human skin [3]. Decorin is a $118 \mathrm{kd}$ proteoglycan (DSPG-II) that affects fibroblasts by arresting proliferation, slowing their migration and reducing their ability to contract collagen gels $[4,5]$. It interacts with TGF- $\beta 1$ to modify its responses in both normal and injured skin [6].

Decorin is available commercially both of bovine and of human origin, but is very expensive. Engel and Catchpole's procedure [1], based on that of Toole and Lowther [2], has routinely been used to recover the inhibitory principle, but it is labour-intensive, time-consuming, and sometimes hazardous because it involves the cooling of a Culatti mill with liquid nitrogen. Most extracts, namely, $\mathrm{A}, \mathrm{B}, \mathrm{C}$, and $\mathrm{E}$, have been of little interest in wound healing, with only $\mathrm{D}$ being of value. We therefore tried to procure extract D more quickly.

There are many other methods for extracting decorin, and skin has been a less regularly used source than other human tissue (eg, articular cartilage [7]), as well as cartilage and muscle from bovine species $[8,9]$. These methods have used extraction procedures that could be quickly adapted to ion-exchange columns, gel chromatography, and density gradient centrifugations for qualitative studies. The use of $4 \mathrm{M}$ guanidine hydrochloride in articular cartilage is a standard procedure http://bssv01.lancs.ac.uk/gig/pages/pg/smallpgs.htm, but it should be noted that many of these procedures for identification and characterization of decorin in qualitative terms are not specifically geared to recovering sufficient yields of native decorin for application in clinical studies. The problem of species differences arises, although decorin from all these sources seems to have similar inhibitory capacities (see below). With regard to the original system of Toole and Lowther [2], the operating costs in terms of time were high, but materials were relatively inexpensive. With these considerations in mind, our main objective has been not only to speed up extraction, but also to retain adequate specific activity of the decorin-enriched extract $\mathrm{D}$, while at the same time obtaining yields from fresh human skin that could potentially be used in clinical application and not just for 


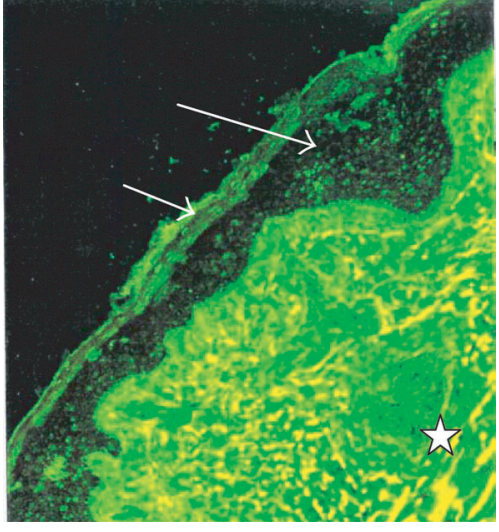

FiguRE 1. Immunofluorescence micrograph of a section of human skin stained with FITC using anti-decorin as the primary antibody (see "materials and methods"). The epidermis falls into two parts, with the outer more cornified cells showing some nonspecific labelling (small arrow), whereas the main part of the epidermis is essentially devoid of staining (large arrow). The dermis is well delineated and is progressively richer in decorin towards its internal aspect (star). Original magnification $\times 240$.

molecular characterisation. Since this paper concerns procedural matters, the "materials and methods" section will be dealt with up front.

\section{MATERIALS AND METHODS}

Skin was obtained at operations of normal female patients undergoing either breast reduction or abdominoplasty, with 10 patients being involved, each having given their consent for the operation and the use of their skin in this specific research project. Full ethical permission was obtained from the local committee for this work. The average age was 35 years, ranging from 25 to 67 . After the operation, the skin was placed in a sterile container and cooled to refrigeration temperature before being collected and used. The time lapse was usually almost 60 minutes, although on 3 occasions it was overnight (16-20 hours) without significant loss of biological activity.

\section{Step 1}

The pieces of skin varied from about 9 to $40 \mathrm{~g}$, a suitable size to handle for each preparation being of almost $20 \mathrm{~g}$ wet weight. They were washed free of blood in icecold isotonic saline before and after being pinned out, epidermal side down, on a Teflon board set into crushed ice. Fat was removed as quickly as possible, after which the surface of the skin was scraped with a solid metal scalpel, and the dermal material placed in a clean preweighed 200$\mathrm{mL}$ Erlenmeyer flask, this being the richest in decorin (Figure 1). (No attempt was made throughout this work to remove the epidermis. Since it is deficient in decorin, it was not worthwhile spending time removing it at the start of the preparation.) The remaining skin was horizontally scored into fine strips with a disposable " $\mathrm{C}$ " blade scalpel,

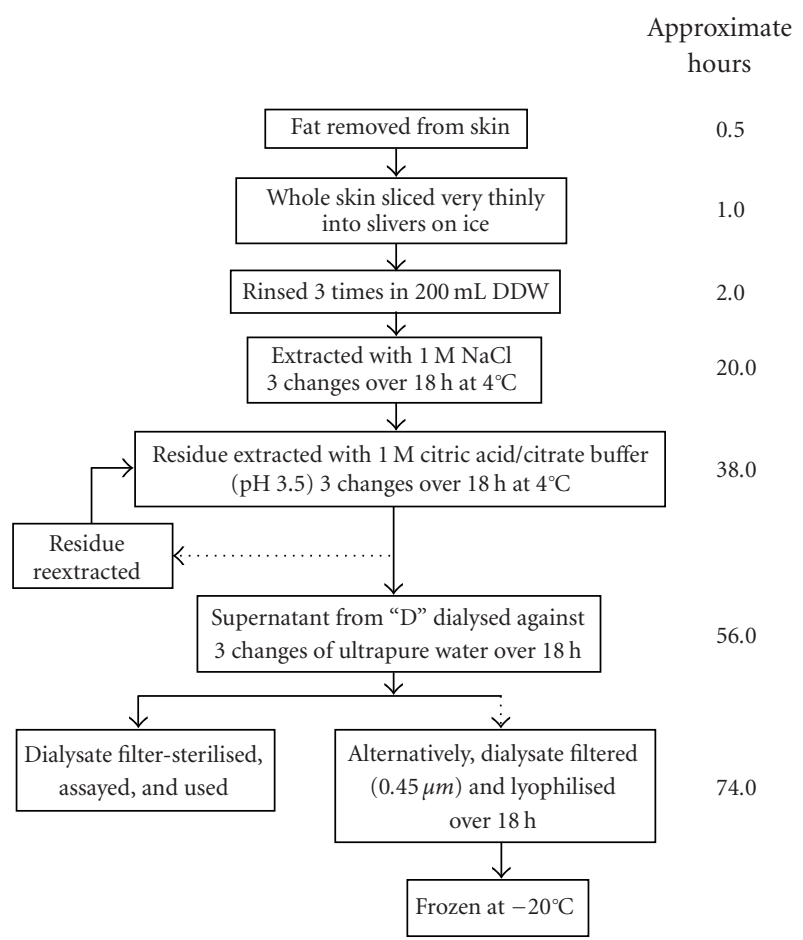

Schema 1. Preparation of human dermal extract D.

followed by slicing at 90 degrees to release thin slivers of skin. The slivers were put into the Erlenmeyer flask, which was reweighed to give the final amount of skin to be extracted. One hundred $\mathrm{mL}$ of ice-cold water was added and the material swirled for 10 minutes; this process was repeated twice. This was followed by a similar operation with ice-cold $0.15 \mathrm{M}$ saline before the skin material was collected on a wire mesh.

The procedure thereafter followed the flow diagram given in Schema 1, and the following steps (see below) indicate most of the important modifications used in reaching this final protocol after many trials. The conventional method of extract D preparation by Toole and Lowther [2] took at least 11 days, whereas the present procedure can reach the same stage in 56 hours without the yield of decorin being substantially reduced.

\section{Step 2}

Skin material was put into $100 \mathrm{~mL}$ ice-cold $1 \mathrm{M} \mathrm{NaCl}$ to which $0.5 \mathrm{~mL}$ saturated phenylmethylsulphonylfluoride solution was added (PMSF, Sigma, Poole Dorset, UK) (but not on all occasions), and the flask swirled at $4^{\circ} \mathrm{C}$ for 18 hours, with changes at 1 hour and 3 to 4 hours.

\section{Step 3}

The skin pieces were collected in a mesh and pressed to remove the salt solution before being placed in 100 $\mathrm{mL} 1 \mathrm{M}$ citrate buffer ( $\mathrm{pH} 3.5$ ) to which PMSF had been added as above. The flask was swirled at $4^{\circ} \mathrm{C}$ for 18 hours, at which time the material was separated into solid and 
supernatant fractions by passing the preparation through a fine steel mesh. The latter was called extract D. The solid residuum was taken once again through Step 3, the second extract being DD, the third DDD, and so on.

\section{Step 4}

Supernatant from Step 3 was put into 5 dialysis bags each containing almost $20 \mathrm{~mL}$ extract, and dialysed against three changes of $5 \mathrm{~L}$ dialysand-using a variety of different solutions for comparison of efficacy-for not less than 20 hours total.

\section{Step 5}

The dialysed extract was put through a $0.45 \mu$ Sartorius filter, set out in $20 \mathrm{~mL}$ aliquots in preweighed sterile containers some was set aside for immediate assay of protein and gel electrophoresis, while the remainder was freeze-dried. The latter material was kept in at $-20^{\circ} \mathrm{C}$ until required, and then reconstituted in a medium without serum to the desired concentration to treat cultures (see below), or in water for protein assay, electrophoresis, and other analyses.

\section{Cell culture}

Human diploid fibroblasts growing in RPMI 1640 with $10 \%$ fetal calf serum were used for growth studies. They were from several different patients (given by different initials-SMIF, JMF, and RWF), although comparison was also made with a fetal foreskin culture, FF/9. A frozen bank of each culture was kept at passage 6 and used within one or two subcultures from this stock in each case.

Cells were seeded at 50000 cells per well in 12-well plates. Growth was followed by two procedures as described previously [3], either trypsinization followed by dispersal and counting electronically in a Coulter counter, or daily estimates of cells by direct phase-contrast microscopy within a designated set of 6 squares on the bottom of each dish. For this latter procedure, cells were normally set out in $25 \mathrm{~cm}^{2}$ flasks.

\section{Other procedures}

Lyophilised extract D was dissolved in water before being adjusted to normal medium strength by the addition of one-tenth volume of 10 times concentrated RPMI 1640 solution. The solution was filter-sterilised through a $0.45 \mu \mathrm{m}$ Sartorius filter and introduced at the required volume to cultures of known protein content ( $\mu \mathrm{g}$ per mL).

Electrophoresis of samples was undertaken precisely as described in our previous paper using a conventional silver staining technique to show up the weak bands [4]. Immunochemical staining for decorin was carried out on 6 to $7 \mu \mathrm{m}$ frozen skin sections stained with antidecorin ( $1: 5$ dilution) as primary, FITC-conjugated anti-rabbit polyclonal antibody ( $1: 20$ dilution) as secondary, as described in [10].

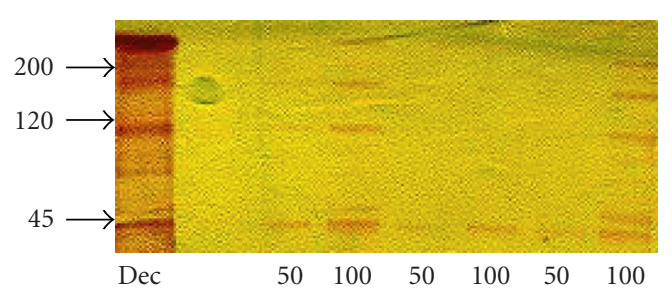

Figure 2. Gel electrophoresis of extracts, as described in "materials and methods" and [4] on 3\%-20\% gradient gels, with $3 \%$ stacking gel. The left lane is a kaleidoscope $\mathrm{M}_{\mathrm{R}}$ marker for decorin with stated values to its left. The lanes are 1 , skin B first extract (D); 2, skin B second extract (DD); 3, skin B third extract (DDD); lane 5 is authentic decorin; lanes $6-8$ are as $2-4$, but for skin C; silver staining.

\section{RESULTS}

\section{Rapid protocol}

After many different variations of the procedure, often as a series on pieces of skin from the same patient or, on other occasions, using pieces from different patients, we slowly moved towards the best way of obtaining adequate amounts of decorin in extract D. The following three criteria were used to assess the product: (a) total soluble protein extracted, (b) its inhibitory activity against fibroblast proliferation in vitro, and (c) the presence of the appropriate bands on gels at 45 and 115-120 kd [4] (Figure 2).

Much of this work will be summarised, with little data from negative or ineffective trials being included where no benefit was gained or a poor yield resulted. Between 15 and 20 separate trials were completed, usually giving a progressively better performance. In many cases, skin specimens were divided into two parts, one going through the best extraction procedure to date and the other being subject to the next modification of the protocol. In one experiment, a 90-g piece of skin was divided into 3 approximately equivalent parts for separate extractions by the latest protocol to check the consistency of the procedure. The preparations were almost identical in every respect, except for a slightly smaller yield from the first piece, indicating that the extraction procedure was relatively reliable and consistent (Figure 3a).

\section{General remarks}

The following annotations give some guidance to the series of experiments that lead to the rapid extraction procedure.

Sterility was not strictly adhered to throughout the procedures. However, careful measures included to minimise the risk were keeping skin cold from the time of collection, undertaking the preparation in a sterile hood, and using ice-cold sterile saline or phosphate buffered saline (PBS) for washing the material 3 times. Sterility of the product at the end of the protocol was ensured by extracts being filter-sterilised before use on cells for testing their inhibitory activity. 


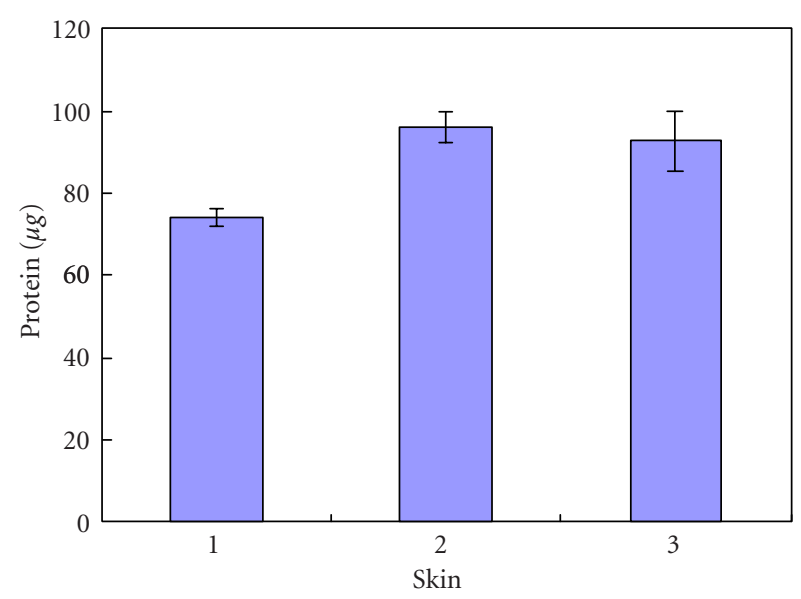

(a)

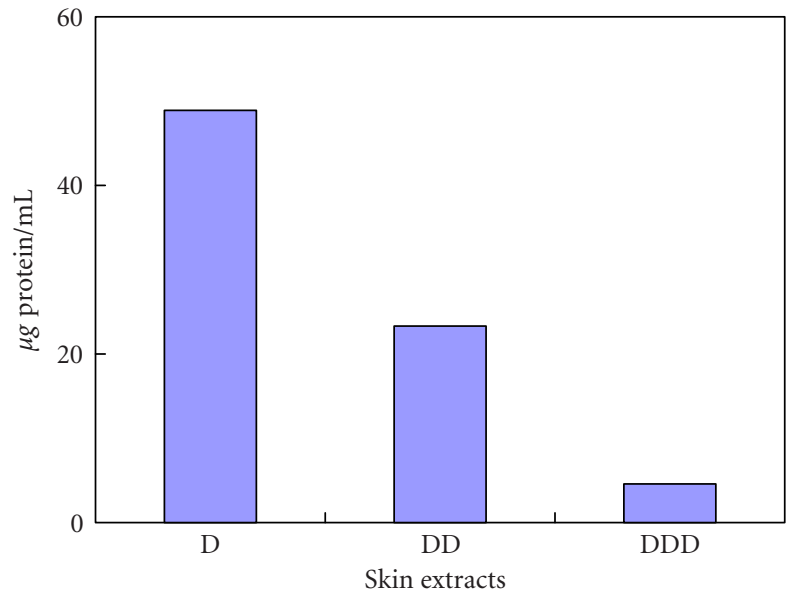

(b)

FIGURE 3. Yields in microgrammes of protein from 3 separate pieces of the same sample of human skin showing the consistent recovery of protein in extract $\mathrm{D}$, each piece having a wet weight of almost $13 \mathrm{~g}$. The bars show the mean $\pm 1 \mathrm{SD}$ of the average protein estimate of 4 determinations.

TABLE 1. Recovery of proteins in extracts from three separate pieces of the same skin treated in identical manner.

\begin{tabular}{lcccc}
\hline Skin & $\begin{array}{c}\text { Wet weight } \\
(\mathrm{mg})\end{array}$ & $\begin{array}{c}\text { Protein in extract } \\
\mathrm{D}(\mathrm{mg})\end{array}$ & $\begin{array}{c}\text { Protein in extract } \\
\text { DD }(\mathrm{mg})\end{array}$ & $\begin{array}{c}\text { Protein in extract } \\
\text { DDD }(\mathrm{mg})\end{array}$ \\
\hline Sample 1 & 13900 & 10.4 & 6.6 & 2.4 \\
Sample 2 & 14200 & $(7.1)^{*}$ & 7.2 & 2.7 \\
Sample 3 & 12400 & 13.0 & 7.8 & 1.9 \\
Average \pm 1 SD & $13500 \pm 960$ & 11.7 & $7.2 \pm 0.6$ & $2.3 \pm 0.3$ \\
Protein in $\mathrm{mg} / \mathrm{g}$ skin & - & 0.87 & 0.37 & 0.17 (total = 1.41$)$ \\
$* *$ Estimated decorin content $(\mu \mathrm{g}) / \mathrm{g}$ skin & - & 78 & 33 & 19 (total = 130) \\
\hline
\end{tabular}

* Some extract accidentally lost during preparation.

**Assuming almost $9 \%$ of mass is decorin (see text).

PMSF was added in the first 7-8 skin preparations. Gels analysed for smearing following procedures with and without PMSF showed no detectable difference, and yields were also unaffected. Activity of the extracts against fibroblast was no different whether PMSF had been used or not. Since PMSF has noxious properties above the concentration we used (ie, a 200-fold dilution of a saturated solution in water), it was deemed safer to leave it out.

Early extractions were simplified over a period of 4 separate preparative runs. The initial skin washings were important to free blood and other unwanted material for the preparations. Extractions in water and normal saline $(0.15 \mathrm{M} \mathrm{NaCl})$ - which yielded extracts $\mathrm{A}$ and $\mathrm{B}$ in the original procedure-were eventually reduced to about half an hour each without much noticeable effect on the protein concentration or activity of extract $\mathrm{D}$ recovered. It seemed that the quicker the pieces of skin reached $1 \mathrm{M}$ $\mathrm{NaCl}$ for extraction overnight the better, although leaving out these earlier steps altogether was not entirely satisfactory. The overnight extraction with $1 \mathrm{~N} \mathrm{NaCl}$ was necessary to remove much collagen and unnecessary proteina- ceous material, and was an important step that could not be omitted without considerable loss of recovery of good specific activity decorin. However, a time-course analysis should, for optimal efficacy, be how long $1 \mathrm{~N} \mathrm{NaCl}$ extraction still needs to be carefully completed. The second overnight extraction with citrate buffer at $\mathrm{pH} 3.5$ was the crucial step.

Repeated extraction with citrate buffer answered the question as to whether a single extraction period of almost 20 hours released significant amounts of or most of the decorin. In brief, the initial overnight extraction with citrate buffer was sometimes almost as good as the original method of Toole and Lowther [2], but we found that skin (the second column of Figure $3 \mathrm{a}$ ) yielded further decorin on the second and third extraction cycles (Figure 3b), although the yield was considerably reduced each time. To maximise release at the second and third extraction cycles, we extended these extraction periods to 72 hours, but it did not significantly increase the overall yield. Details for the sequence of extractions have also been summarised in Table 1. The second and third extracts (DD and DDD) 


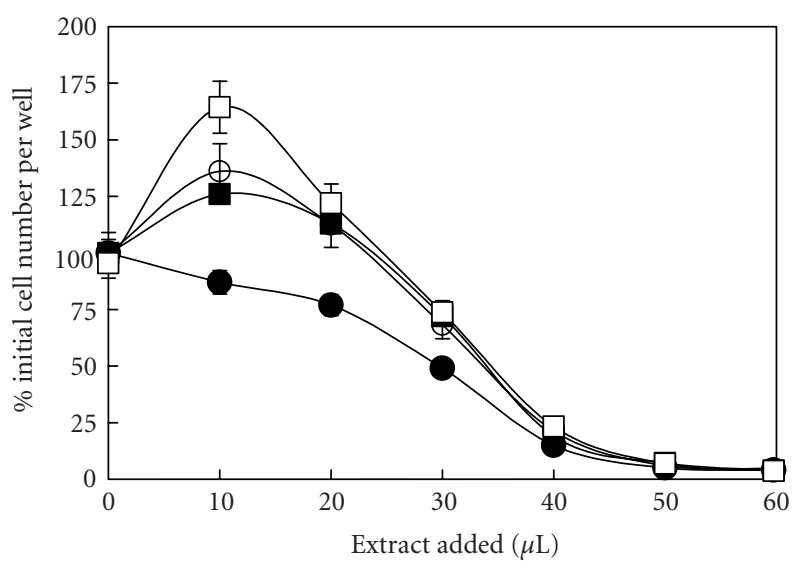

FIgURE 4. Graphs showing the ability of the three skin extracts $\mathrm{D}$ shown in Figure 3 to inhibit fetal fibroblasts (FF) growth. The assay was made after 3 days growth and records the increase in cell number relative to the control ( $100 \%$ at 0 extract). The amount of extract represented in the added volumes was $\sim 50$ $\mu \mathrm{g}$ per $\mathrm{mL}$. The symbols refer to: $\square$ extract $1 ; \mathbf{\square}$ extract 2 ; 0 extract 3 ; $\bullet$ pure decorin. The values are means of 4-6 determinations at each point, \pm 1 SD. (Where bars are not evident, they lie within the symbols.) Note that at low concentration, decorin has no stimulatory effect on cell growth.

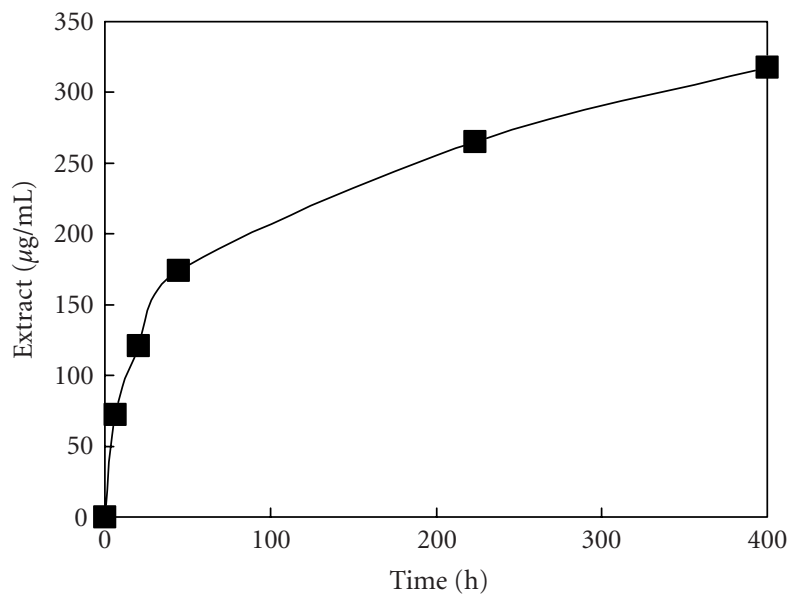

FIgURE 5. Release of extract D from human skin following a prolonged incubation with a single batch of the citrate buffer. The curve demonstrates the protein recovery, which is related closely with its antifibroblast activity. More than half the maximal extraction at 400 hours was achieved within the first 60 hours. Time points are single values taken from a single extraction procedure.

also gave very similar fibroblast inhibition on a specific activity basis, but one notable difference was the slightly higher stimulatory effect of the first extract (extract D) compared with the second and third extracts (Figure 4). A continuous extraction for more than 2 weeks was undertaken (Figure 5), without the extraction buffer being changed throughout this period. This showed that rapid

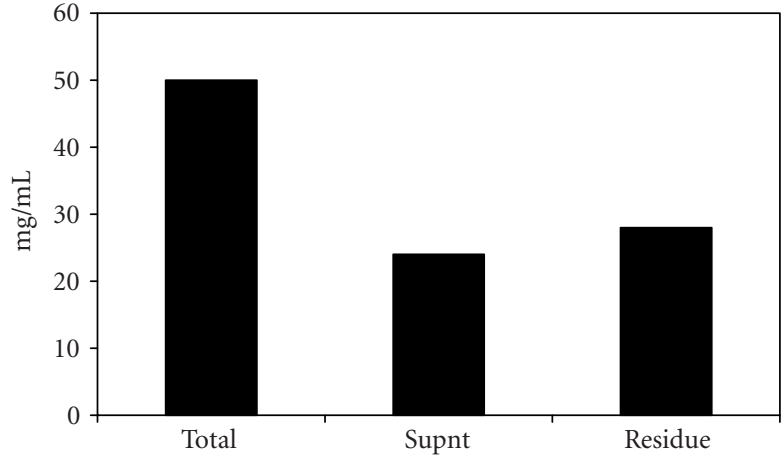

FIgURE 6. Total extract D from an incubation using the short protocol. The extract was dialysed in $0.15 \mathrm{~N} \mathrm{NaCl}$, and the protein recovery measured in the supernatant (supnt) and in the material that precipitated out during the dialysis (residue). These both retained decorin-like activity, as shown in Figure 7.

extraction occurred for 50 to 60 hours, but thereafter accumulation of active extract was much slower. Indeed, between a third and a half of the maximum extraction was released with an 18-20 hour single overnight extraction, if a compromise between yield and extraction time is sought.

Dialysis was carried out against a range of different dialysing solutions to see whether this improved recovery. Saline, PBS, and water, with or without EDTA $(0.1 \mathrm{mM})$, have all been used. In brief, water proved the best dialysand, as in the original method. Addition of EDTA made no difference. When the $\mathrm{pH}$ remained below 4.2 in a salt-containing dialysand, a flocculent precipitate invariably developed comprising about half the total protein of extract D (Figure 6). This was removed by filtration through a $0.2 \mu \mathrm{M}$ filter, and the filtrate adjusted to neutrality with a small amount of $1 \mathrm{~N} \mathrm{NaOH}$. The residuum was resuspended in water and adjusted to neutrality, which resulted in all but $8 \%-14 \%$ of the total protein redissolving (see Table 1). Analysis of the two fractions on the fibroblast proliferation inhibition assay showed that both had similar specific activities in their inhibitory action on fibroblasts (ie, per $\mu$ g protein; Figure 7).

Water was in the end preferred for dialysis; a $20 \mathrm{~mL}$ volume of extract $\mathrm{D}$ dialysate increased to $23 \mathrm{~mL}$, with negligible contamination with salts. Dialysis against $0.15 \mathrm{~N} \mathrm{NaCl}$, in comparison, increased the volume of extract $\mathrm{D}$ to barely $21.3 \mathrm{~mL}$, with the salt content equilibrating at almost $12 \mathrm{mg} / \mathrm{mL}$, but with much heavier precipitation of protein that was more difficult to redissolve when the $\mathrm{pH}$ was adjusted to neutrality. The lack of salt in the former dialysate meant lyophilisation gave an extremely small residue of dirty whitish (sometimes slightly brownish) fluffy "glass," which readily redissolved in water or any other medium at the desired concentration. Lyophilisation of extract with salts in the dialysand gave a voluminous white residue that readily redissolved in aqueous solution. 


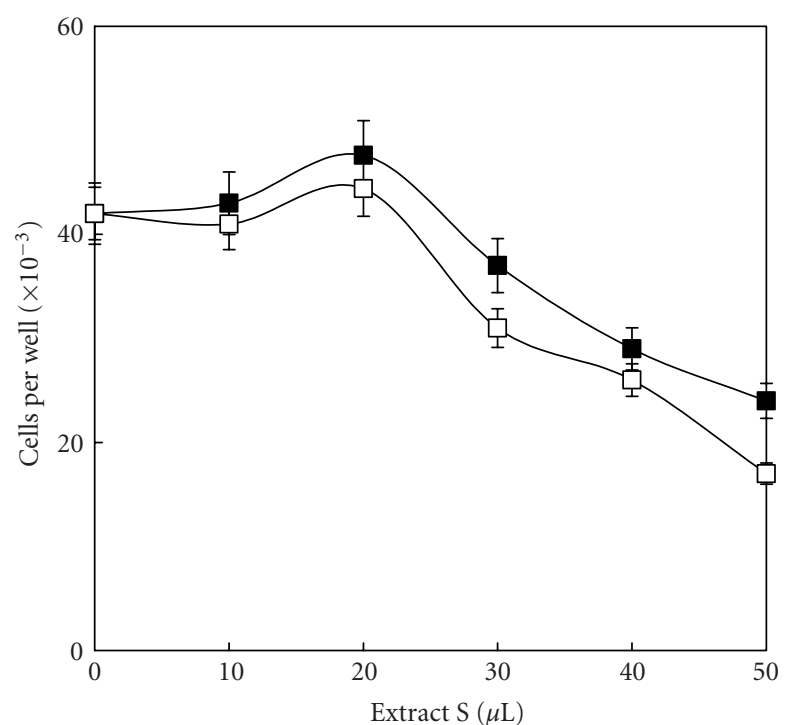

Figure 7. Inhibition of fibroblast growth by extract as supernatant $(\square)$ and residue ( $\square$ ), as shown in Figure 6. The values are means of $4-6$ determinations per time point \pm 1 SD.

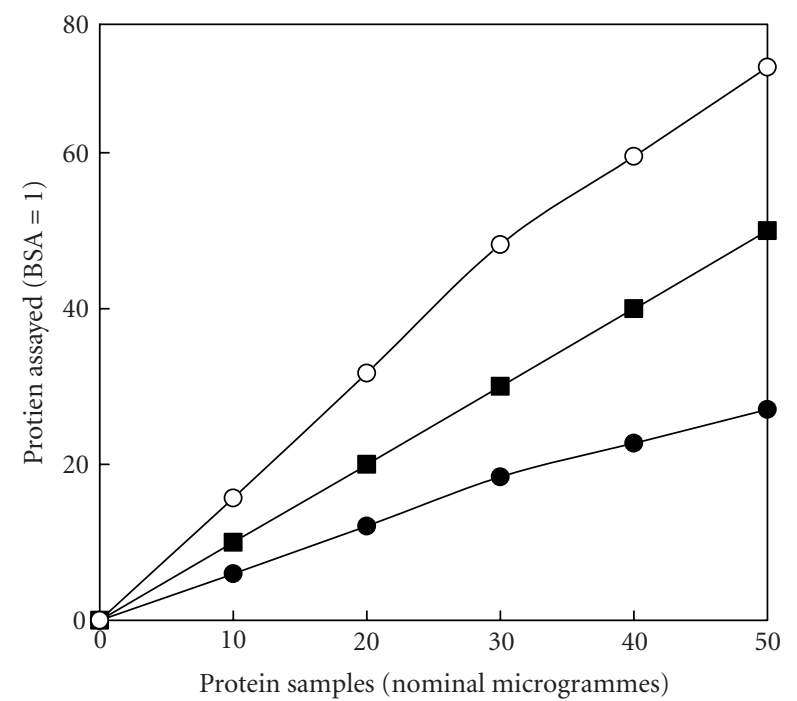

Figure 8. Lowry's analysis of preparations made on a mass basis (nominally taken as protein) and plotted against the colorimetric assay. Bovine serum albumin (BSA) is taken as a standard and is plotted as a straight-line correlation of $1(\boldsymbol{\square})$. Authentic decorin $(\bullet)$ is considerably below this line, indicating that its mass as a proteoglycan is underestimated by the Lowry assay. On the basis of protein relative to glycosaminoglycan in decorin (ie, $118 / 45 \mathrm{kd}$ ), the curve ( $\mathrm{O}$ ) has been plotted, which now indicates an overshoot in estimation. Hence by inference, the preparation will be in core protein rather than the full-size proteoglycan (see Figure 3).

Protein recovery for extract $\mathrm{D}$ was usually in the range of $0.75-1.5 \mathrm{mg}$ per gram of skin (Table 1 ; cf [7]). However, the actual mass of decorin molecules could have been underestimated by $60 \%-70 \%$ by Lowry's analysis because of the relative size of the glycosaminoglycan side chain

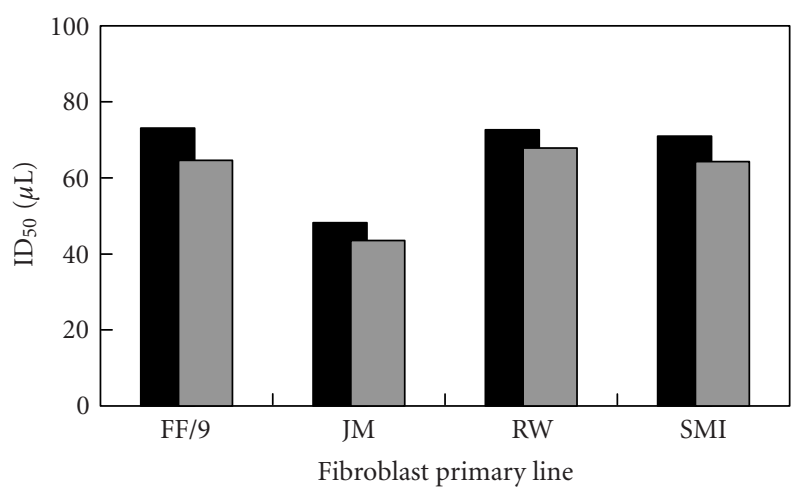

Figure 9. Effect of an extract D preparation on 4 different human fibroblast primary cultures, three from adult patients (JM, RW, and SMI) and one culture of fetal fibroblasts (FF). The bars plot the $\mathrm{ID}_{50}$ in terms of the volume in microlitres of extract added, each having been prepared to the same mg per $\mathrm{mL}$ prior to the start of an incubation for 3 days before estimation. The dark bars show extract from the rapid extraction procedure, and the grey bars show extract from the old procedure. The variation in sensitivity of cell lines around mean $\mathrm{ID}_{50}$ of approximately $60 \mu \mathrm{L}$ is relatively small, although JM fibroblasts were clearly more sensitive to both preparations.

(Figure 8) that does not contribute to the protein assay. The exact amount of decorin cannot therefore be determined easily and directly simply by measuring protein yield. Our estimation was carried out biologically, on the basis of relative inhibitory activity against a bovine articular decorin standard (almost $88 \%$ pure) from Sigma. It assumed no stimulant effect of extract D having to be offset by its decorin content, and yet clearly it can be seen from Figure 8 that this also gives an underestimate. The decorin yield averaged $9 \%$ of the total yield, with a range of from almost 5 to a maximum of $12 \%-13 \%$ (Table 1 ).

Lowry's analysis of several simultaneous preparations showed that in a typical preparation of each type, the original method yielded an extract with more protein (almost $70 \mu \mathrm{g} / \mathrm{mL}$ ), whereas our rapid extraction yielded less (almost $45-50 \mu \mathrm{g} / \mathrm{mL}$ ). In terms of inhibitory activity, as seen from Figure 9, the rapid extraction method gave a consistently weaker response over three days of inhibition, assessed on the $\mathrm{IC}_{50}$ at this time on the four different batches of human fibroblasts tested, and the two procedures were not at variance by more than $10 \%-15 \%$ in total yield of active compound. Therefore, in terms of overall recovery of decorin, the compromise was about $20 \%-30 \%$ on the original procedure for a saving of some 8 days or more in recovery time.

\section{DISCUSSION}

The rapid extraction process usually yields less protein but gives a better dose response curve, suggesting that the rapid extraction process is being slightly more selective for the active compound, or possibly that speeding up the overall process preserves more active molecules than the 
old methods $[1,2]$. It has been impossible to estimate loss of activity during preparation, but it is significant and is unlikely to be due to proteolysis, since inclusion of PMSF throughout the process made no significant difference to recovery, and was therefore omitted. Keeping preparations cool was enough to maintain activity of decorin, which is a relatively stable molecule.

The new extraction procedure is not radically different from the original one, but essentially leaves out unnecessary steps or reduces them to the shortest convenient time. However, the exercise was necessary to show that it could be done quickly without totally compromising the recovery of a decorin-enriched fraction, and also to test its reliability and reproducibility (Figures 3 and 9). From the re-extraction work (Figure 5), it appears that a continuous automated recovery protocol could be adopted to leach out as much decorin as possible over a period of about a week where time is no object.

The product can be lyophilised and readily redissolved in water, PBS, or any other medium in order to treat cultures. A slight enhancement of fibroblast growth was consistently seen at low concentrations, but has never once been found with "pure" decorin preparations. It follows that the stronger stimulatory effect at low doses is due to an impurity that operates at low concentration on cells, and that its effect must be overridden by decorin itself as concentration increases. Therefore if our decorin-rich extract were further purified to free itself of the stimulant, it would show a slightly higher inhibitory action than indicated from our present data.

We have not regularly taken our preparations through further purification here, but continuous extraction followed by fractionation as described in [4] on a preparative scale will yield a considerable amount of high-activity decorin from human skin. The amount of decorin is considerable when one considers that its main function is probably to repress the action of TGF- $\beta$. Decorin is a lowaffinity high-capacity inhibitor in this respect, but the evidence suggests that it may have other properties yet to be discovered (see below).

Comparison of our preparation has so far been made against bovine decorin recovered at a purity of almost $88 \%$ from articular cartilage. This may not be a fair comparison with the human skin proteoglycan, since the core protein may not be entirely homologous and the glycosaminoglycan chain associated with it might differ. However, both are inhibitory, so there has to be at least a close "physiological" homology. There has also been the need for authentic human decorin as a standard. More recently, EMP Genetech (Denzlingen, Germany) and R\&D Systems Europe Ltd (Abingdon, UK) have marketed $h u$ man recombinant decorin of improved purity (more than $95 \%)$, but at costs that are substantially higher than the Sigma bovine preparation, and these new products have yet to be tested in our laboratory. Once again, these expensive products are financial disincentives to the use of decorin preparations for wound treatment in clinical trials, whereas our method provides a plentiful and cheap source of the natural human product that, applied topically, might well be in clinical use in the near future.

In a following report, we will include comparisons of activities of some of these preparations on the following systems: fibroblasts growth with and without TGF- $\beta$ treatment, melanoma cells, and in vitro angiogenesis. This is in keeping with the clear-cut evidence that decorin might be useful in other theatres of action, for example, in cancer, and not just in wound healing $[11,12]$.

\section{ACKNOWLEDGMENTS}

This work has been supported by funds from the Burns Fund, Grampian University Hospitals Trust, and Action Research. We thank Miss Satvir Minhas for technical assistance.

\section{REFERENCES}

[1] Engel MB, Catchpole HR. Collagen distribution in the rat demonstrated by a specific anti-collagen antiserum. Am J Anat. 1972;134(1):23-39.

[2] Toole BP, Lowther DA. Dermatan sulfate-protein: isolation from and interaction with collagen. Arch Biochem Biophys. 1968;128(3):567-578.

[3] Muir IFK, Padilla-Lamb A, Stewart JE, Wheatley DN. Growth inhibition of cultured fibroblasts by extracts from human dermis. Br J Plast Surg. 1997;50(3):186-193.

[4] Stewart JE, Wheatley DN, Holmes JD, Muir IFK. Purification and identification of a human dermal extract component inhibitory to fibroblast proliferation. Cell Biol Int. 2001;25(7):607-612.

[5] El-Sheemy MA, Muir IFK, Wheatley DN, Eremin O. Inhibition of the contraction of collagen gels by extracts from human dermis. Cell Biol Int. 2001; 25(7):635-642.

[6] De Luca A, Santra M, Baldi A, Giordano A, Iozzo RV. Decorin-induced growth suppression is associated with up-regulation of $\mathrm{p} 21$, an inhibitor of cyclindependent kinases. J Biol Chem. 1996;271(31): 18961-18965.

[7] Roughley PJ, White RJ. Dermatan sulphate proteoglycans of human articular cartilage. The properties of dermatan sulphate proteoglycans I and II. Biochem J. 1989;262(3):823-827.

[8] Rosenberg LC, Choi HU, Tang LH, et al. Isolation of dermatan sulfate proteoglycans from mature bovine articular cartilages. J Biol Chem. 1985;260(10):63046313.

[9] Eggen KH, Malmstrom A, Kolset SO. Decorin and a large dermatan sulfate proteoglycan in bovine striated muscle. Biochim Biophys Acta. 1994;1204(2): 287-297.

[10] Stewart JE. The influence of a natural dermal extract on wound healing. $\mathrm{PhD}$ thesis, University of Aberdeen, Aberdeen, UK, 1998. 
[11] Nash MA, Loercher AE, Freedman RS. In vitro growth inhibition of ovarian cancer cells by decorin: synergism of action between decorin and carboplatin. Cancer Res. 1999;59(24):6192-6196.

[12] Iozzo RV. Heparan sulfate proteoglycans: intricate molecules with intriguing functions. J Clin Invest. 2001;108(2):165-167.

* Corresponding author.

E-mail: info@biomedes.co.uk

Fax: +44 1224 274179; Tel: +44 1224274173 

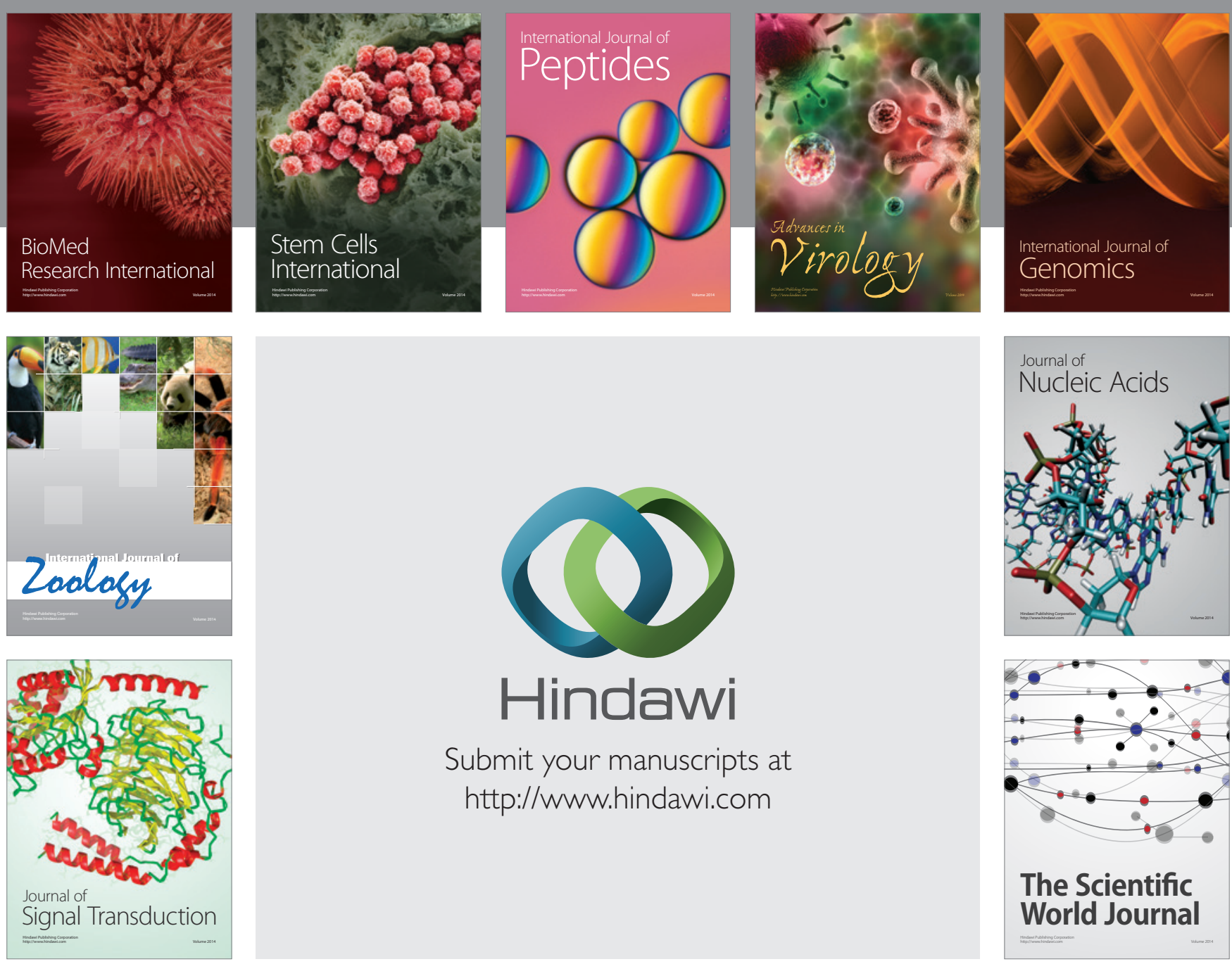

Submit your manuscripts at

http://www.hindawi.com
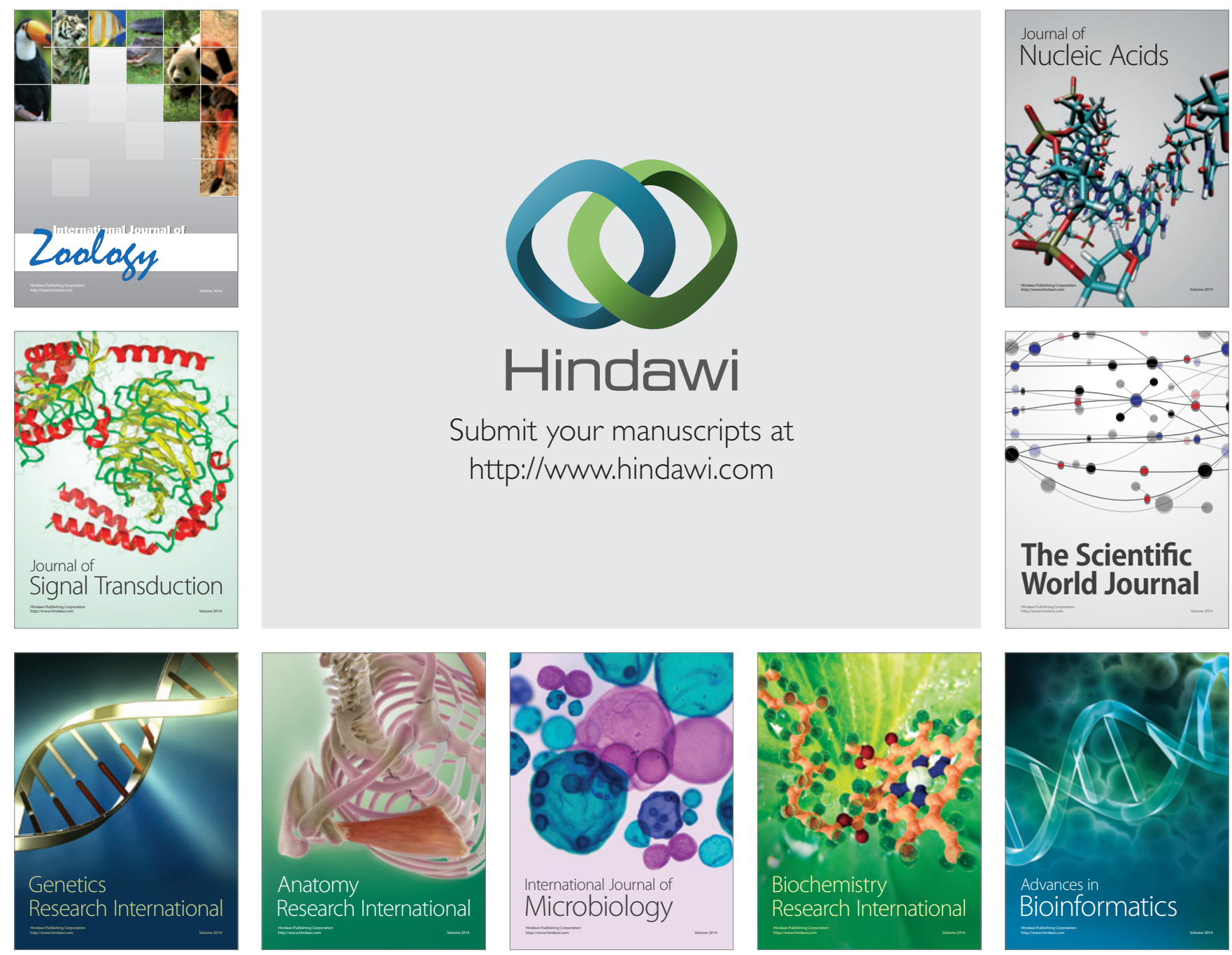

The Scientific World Journal
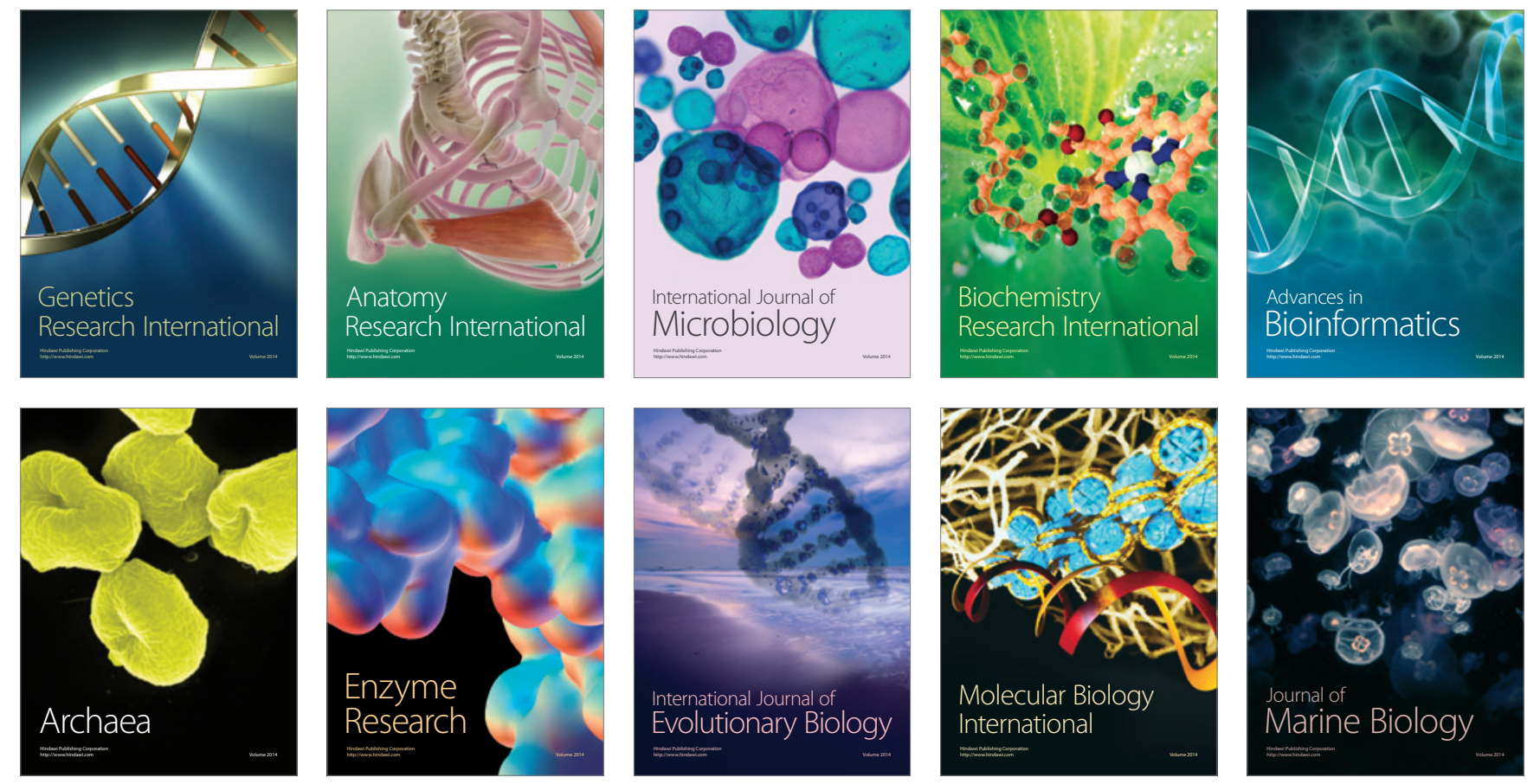